\title{
Uso de Oxitocina para Prevenir Mortalidad Embrionaria Temprana en Alpacas
}

\author{
Use of Oxytocin for the Prevention of Early Embryonic Mortality in Alpacas
}

Silvia Sánchez B. ${ }^{1,3}$, Víctor Leyva $V_{\bullet}{ }^{1,4}$, Alex Oscanoa M. ${ }^{1}$, Wilber García V. ${ }^{2}$

\section{Resumen}

El objetivo del presente experimento fue comprobar el efecto de la oxitocina en la supervivencia embrionaria durante el periodo de riesgo por luteolisis en alpacas posservicio. Se seleccionaron 43 alpacas adultas receptivas al macho y con un folículo ovárico e» $7 \mathrm{~mm}$ de diámetro, que fueron sometidas a monta controlada e» 10 minutos con macho fértil, asegurando la ovulación con $1 \mathrm{ml}$ de GnRH i.m. (Día 0). Dentro de las alpacas con cuerpo lúteo confirmado por ecografía transrectal en el día 7 , se seleccionaron 30 animales al azar y se distribuyeron en dos grupos similares. Al primer grupo se le aplicó $40 \mathrm{UI} / \mathrm{d}$ de oxitocina los días 9,10 y 11 pos-servicio, fraccionadas en 4 dosis de $10 \mathrm{UI}$ cada 5 horas. Al segundo grupo se le aplicó un placebo como control. Se evaluó la supervivencia embrionaria por receptividad sexual los días $8,10,11,12$ y 40 pos-servicio. La viabilidad embrionaria fue confirmada el día 40 pos-servicio mediante ecografía. Las alpacas tratadas con oxitocina tuvieron porcentajes de supervivencia embrionaria de $100 \%$ los días 8 y 10 , de $93.3 \%$ los días 11 y 12 , y de $86.7 \%$ el día 40 pos-servicio, en tanto que las alpacas no tratadas fueron de $100,80,66.7,46.7$ y $46.7 \%$ los días $8,10,11,12$ y 40 posservicio, respectivamente, habiendo diferencias entre grupos los días 12 y $40(p<0.05)$. Se concluye que la oxitocina exógena redujo la mortalidad embrionaria en alpacas antes de los 40 días de gestación.

Palabras clave: oxitocina; mortalidad embrionaria; luteólisis; alpacas

${ }^{1}$ Laboratorio de Reproducción Animal, Facultad de Medicina Veterinaria, Universidad Nacional Mayor de San Marcos, Lima, Perú

${ }^{2}$ Estación Experimental del Centro de Investigación IVITA-Maranganí, Universidad Nacional Mayor de San Marcos, Cusco, Perú

${ }^{3}$ E-mail: crisbasisa@gmail.com

${ }^{4}$ E-mail: vleyva@unmsm.edu.pe

Recibido: 12 abril de 2017

Aceptado para publicación: 15 de junio de 2017 
The objective of the present experiment was to test the oxytocin effect on embryonic survival during the luteolysis risk period in post-mating alpacas. Forty-three adult alpacas that were receptive to the male and bearing an ovarian follicle $\geq 7 \mathrm{~mm}$ of diameter were selected. They were mated with fertile males and the copula lasted for $\geq 10$ minutes. Ovulation was secured with con $1 \mathrm{ml}$ of GnRH i.m. (Day 0). Within the group of alpacas with a corpus luteum confirmed by trans-rectal echography on day 7, 30 were randomly selected and assigned into two similar groups. One group received 40 IU oxitocin on days 9,10 and 11 post-mating days distributed in 4 doses of 10 IU every 5 hours. The second group was considered as control. The embryonic survival was evaluated based on sexual receptivity on days $8,10,11,12$ and 40 post-mating and confirmed on day 40 by ultrasonography. The oxytocin treated alpacas resulted with embryonic survival of $100 \%$ on days 8 and $10,93.3 \%$ on days 11 and 12 , and $86.7 \%$ on day 40 after mating, whereas non-treated alpacas had 100, 80, 66.7, 46.7 and $46.7 \%$, on days $8,10,11,12$ and 40 after mating respectively, with significant differences between groups on days 12 and 40 $(p<0.05)$. In conclusion, exogenous oxytocin reduced the embryonic mortality in alpacas before 40 days of pregnancy.

Key words: embryonic mortality; oxytocin; luteolysis; alpaca

\section{INTRODUCCIÓN}

El 50\% de la mortalidad embrionaria en alpacas ocurre antes de los 35 días de gestación (Fernández-Baca, 1970). Estudios posteriores demostraron que un $12 \%$ de esta mortalidad ocurre dentro de los 5 días posovulación, posiblemente debido a la luteolisis causada por la presencia de folículos estrogénicos (Leyva y García, 2000).

La ovulación en los camélidos es inducida por el coito, y el tamaño folicular en la alpaca es mayor a $6 \mathrm{~mm}$ (Bravo y Sumar, 1989), con alta concentración de estradiol vinculado a la actividad folicular (Bravo et al., 1991; Aba et al., 1995), y sobretodo asociada a la descarga de la hormona luteinizante (LH) (Fernández-Baca, 1970). En alpacas y llamas, folículos mayores de $7 \mathrm{~mm}$ están directamente relacionados a una mayor descarga de LH, y por consiguiente a la ovulación y formación de cuerpo lúteo (Bravo et al., 1988). La actividad secretora de progesterona del cuerpo lúteo es esencial para el mantenimiento de la preñez (Leyva y García, 1999a), ocasionando el rechazo del macho 5 días después de la ovulación (Fernández-Baca, 1971).

La luteolisis en alpacas ocurre entre el día 9 y 12 pos-ovulación (Fernández-Baca, 1970). La prostanglandina $\mathrm{F}_{2 \alpha}\left(\mathrm{PGF}_{2 \alpha}\right)$ es secretada por el endometrio y está considerada como el agente luteolítico en camélidos sudamericanos, aunque no se conoce su mecanismo de acción (Leyva y García, 1999a). En la vaca y oveja, el estradiol actúa sobre las células endometriales, estimulando la aparición de receptores de oxitocina, y esta hormona a su vez estimula la secreción de $\mathrm{PGF}_{2 \alpha}$ (McCracken, 1984). La concentración de progesterona en sangre se eleva desde el día 4 pos-servicio alcanzando su máximo el día 8 y $9(2.2 \mathrm{ng} / \mathrm{ml})$. Luego, entre el día 9 y 11 pos-ovulación se produce una disminución temporal de progesterona y, a su vez, la secreción pulsátil y baja de $\mathrm{PGF}_{2 \alpha}$ en alpacas que han llegado a concebir (Aba et al., 1995). Es durante este periodo de riesgo que se presume ocurre el reconocimiento maternal de 
la preñez (Novoa y Leyva, 1996), como ocurre en llamas donde se inicia la regresión luteal inducida por el útero pero es rescatada por el embrión (Adams et al., 1991).

La perfusión intrauterina de proteína trofoblástica obtenida de embriones cultivados de ovinos de 15 a 16 días fue capaz de prolongar el periodo de vida del cuerpo lúteo en ovejas cíclicas (Godkin et al., 1984). En alpacas se ha observado que la administración de estrógenos durante los días 8 y 9 de gestación resultó en mayor supervivencia embrionaria (Chipayo et al., 2003), por lo que se cree que este efecto sobre la preñez en alpacas es similar a lo que ocurre en marranas. El estradiol es producido por el tejido embrionario entre los días 11 y 12 , inhibiendo la luteolisis debido a que induce la mayor descarga de $\mathrm{PGF}_{2 \alpha}$ dentro del lumen uterino impidiendo su acción sobre el cuerpo lúteo (Bazer et al., 1986; Geiser et al., 1994).

La alpaca puede concebir a partir de los 5 días posparto (Sumar et al., 1972), y el el pico de producción de leche ocurre entre los días 8 y 13 de lactación, por lo que se asume una mayor producción de oxitocina en ese periodo (Leyva et al., 1983), pero se desconoce el efecto de esta hormona en la gestación temprana. El objetivo del presente estudio fue determinar el efecto de la administración de oxitocina entre los días 9, 10 y 11 del servicio en alpacas gestantes.

\section{Materiales y Métodos}

El estudio se realizó en mayo y junio de 2007 en el Centro de Investigación y Producción La Raya de la Universidad Nacional del Altiplano, Puno, localizado a una altitud de $4300 \mathrm{msnm}$. Se seleccionaron 43 alpacas hembras vacías mediante ecografía de transducción (Aloka 220, Japón) de un rebaño de 350 alpacas adultas vacías criadas en praderas nativas y sujetas a iguales condiciones de manejo. Las alpacas seleccionadas tenían un folículo pre-ovulatorio $\geq 7 \mathrm{~mm}$ de diámetro y fueron sometidas a monta na- tural con macho fértil. El tiempo de cópula aceptado fue no menor de 10 ni mayor de 20 minutos (Fernández-Baca, 1970). Al término de la monta se administró $1 \mathrm{ml}$ de hormona liberadora de gonadotropinas sintética (GnRH), equivalente a $0.0042 \mathrm{mg} /$ animal de acetato de Buserelina, para asegurar la ovulación y reforzar el efecto lúteotrópico (Leyva y García, 1999b).

La ocurrencia de la ovulación se evaluó el día 7 luego del servicio. De las 33 alpacas que rechazaron al macho y presentaron cuerpo lúteo detectado por ecografía rectal, se distribuyeron 30 alpacas al azar en dos grupos similares. Las hembras del primer grupo recibieron una dosis diaria de $40 \mathrm{UI}$ de oxitocina los días 9, 10 y 11 pos-servicio, fraccionadas en cuatro dosis de 10 UI cada una. En el día 9, las tres primeras dosis se aplicaron cada 5 horas desde las 7 am, vía intramuscular, excepto la última dosis que se aplicó vía subcutánea. En los días 10 y 11 se repitió el mismo régimen de aplicación, pero solo por vía subcutánea. Al segundo grupo se les aplicó un placebo como control siguiendo el mismo protocolo. La gestación temprana se evaluó los días $8,10,11,12$ y 40 a través de receptividad sexual, y en el día 40 mediante ecografía transrectal para observar la vesícula embrionaria.

La tasa de supervivencia embrionaria se evaluó mediante la tabla de contingencia de Chi Cuadrado.

\section{Resultados}

El Cuadro 1 muestra los porcentajes de ovulación, el desarrollo de un cuerpo lúteo y la tasa de supervivencia embrionaria en alpacas antes, durante y después del tratamiento con 40 UI de oxitocina. Se consiguió la ovulación en todas las hembras de ambos grupos, con la consiguiente formación de un cuerpo lúteo. Esto significó que las hembras rechazaran al macho antes del inicio del experimento. 
Cuadro 1. Frecuencia (\%) de supervivencia embrionaria en alpacas tratadas con 40 UI de oxitocina o con placebo los días 9,10 y 11 posservicio

\begin{tabular}{cccc}
\hline $\begin{array}{c}\text { Supervivencia } \\
\text { embrionaria }\end{array}$ & $\begin{array}{c}\text { Oxitocina } \\
(\mathrm{n}=15)\end{array}$ & $\begin{array}{c}\text { Placebo } \\
(\mathrm{n}=15)\end{array}$ & $\begin{array}{c}\text { Total } \\
(\mathrm{n}=30)\end{array}$ \\
\hline 8 & $100.0^{\mathrm{a}}$ & $100.0^{\mathrm{a}}$ & 100.0 \\
10 & $100.0^{\mathrm{a}}$ & $80.0^{\mathrm{a}}$ & 90.0 \\
11 & $93.3^{\mathrm{a}}$ & $66.7^{\mathrm{a}}$ & 80.0 \\
12 & $93.3^{\mathrm{a}}$ & $46.7^{\mathrm{b}}$ & 70.0 \\
40 & $86.7^{\mathrm{a}}$ & $46.7^{\mathrm{b}}$ & 66.7 \\
\hline \multicolumn{4}{c}{ a,b Superíndices diferentes dentro de columnas } \\
indican diferencia estadística ( $\mathrm{p}<0.05)$
\end{tabular}

Se encontró una mayor supervivencia embrionaria en alpacas tratadas que en aquellas del grupo control, habiendo diferencia estadística $(\mathrm{p}<0.05)$ los días 12 y 40 pos-servicio.

\section{Discusión}

Se observó mayor frecuencia de supervivencia embrionaria en alpacas tratadas con oxitocina que en las tratadas con placebo. En la fase luteal de los rumiantes, la oxitocina endógena reacciona en el endometrio con su receptor para la liberación de $\mathrm{PGF}_{2 \alpha}$ (McCracken et al., 1984), lo cual indica su acción como mediador luteolítico(Flinty Sheldrick, 1983). Sin embargo, en el presente estudio, la oxitocina exógena tuvo un efecto contrario, ejerciendo al parecer un efecto lúteotrópico en alpacas con gestación reciente.

Hay algunos reportes que demuestran una mayor vida útil del cuerpo lúteo con la administración de oxitocina en vaquillas (Tallam et al., 2000), vacas (Gilbert et al., 1989) y ovejas (Flint y Sheldrick, 1985). Un primer mecanismo probable de acción de la oxitocina exógena parece estar relacionada al agotamiento de los receptores endometriales, bloqueando la acción de la oxitocina endógena, y por consiguiente disminuyendo la liberación de PGF $_{2 \alpha}$ (Sheldrick y Flint, 1990). Esto, a su vez, ocasiona la permanencia del cuerpo lúteo y la liberación de progesterona, hormona que limita el desarrollo del folículo dominante productor de estradiol, que es la hormona encargada de sintetizar los receptores endometriales para la oxitocina (Hixon y Flint, 1987; Vallet et al., 1990).

Un segundo mecanismo probable de acción de la oxitocina exógena sería sobre su acción en las células luteales. La oxitocina es secretada por las células luteales grandes que son las que producen progesterona (Rodgers et al., 1983a) y que, además, tienen receptores de LH (Fitz et al., 1982). Las células luteales pequeñas también poseen receptores de $\mathrm{LH}$, reaccionando al estímulo con la liberación de progesterona (Rodgers et al., 1983b). Estos datos sugieren que el contacto entre células luteales grandes y pequeñas puede ser importante para la respuesta a la oxitocina exógena (Walters et al., 1984).

Un tercer mecanismo que explique la acción de la oxitocina exógena puede ser debido a su potente efecto liberador de estradiol proveniente de las células luteales pequeñas; el estradiol a su vez, estimula la liberación de progesterona (Jarry et al., 1990). En cerdas se ha demostrado que el estradiol de origen fetal bloquea el efecto luteolítico de la $\mathrm{PGF}_{2 \alpha}$, asegurando la permanencia del cuerpo lúteo y por lo tanto de la preñez (Geiser et al., 1994). La administración de estradiol en alpacas durante los días 8 y 9 de gestación resultó en mayor supervivencia embrionaria (Chipayo et al., 2003), por lo que se sugiere la hipótesis de un efecto lúteotrópico de la oxitocina a través de la regulación de los niveles de progesterona y estradiol (Liebermann y Schams, 1995). 
En alpacas vacías con ovulación inducida, la luteolisis ocurre en el día 11 después del servicio (Leyva y García, 1999c), en tanto que en alpacas gestantes, los niveles de progesterona disminuyen entre el día 10 y 11 después del servicio (Aba et al., 1995). Se asume que es durante este periodo de 48 horas de disminución de progesterona que ocurre el reconocimiento maternal de la preñez por efecto del estradiol de origen fetal (Chipayo et al., 2003). Además, el riesgo de luteolisis aumenta durante este periodo, probablemente debido a una alteración neuroendocrina ocasionada por factores medioambientales y de manejo y que, en consecuencia, pueden resultar en mortalidad embrionaria (Leyva y García, 2000).

En las alpacas, el empadre se realiza después del día 10 posparto, coincidiendo con una alta producción de leche y, por lo tanto, de una mayor producción de oxitocina (Leyva et al., 1983). En este caso, la oxitocina puede actuar favoreciendo la gestación mediante una regulación de las hormonas progesterona y estradiol. En el presente experimento, la oxitocina exógena aplicada en los días 9,10 y 11 pos-servicio redujo la mortalidad embrionaria sugiriendo un efecto lúteotrópico. No se sabe si este efecto es debido al aumento de progesterona, de estradiol, o de ambos, o quizás debido también a la disminución de $\mathrm{PGF}_{2 \alpha}$, lo cual deberá ser demostrado en posteriores ensayos.

\section{Literatura Citada}

1. Aba M, Forsberg M, Kindahl H, Sumar J, Edqvist L. 1995. Endocrine changes after mating in pregnant and non-pregnant llamas and alpacas. Act Vet Scand 36: 489-498.

2. Adams G, Sumar J, Ginther O. 1991. Form and function of the corpus luteum in llamas. Anim Reprod Sci 24: 127-138. doi: 10.1016/0378-4320(91)90088-H

3. Bazer F, Vallet J, Roberts R, Sharp D, Thatcher W. 1986. Role of conceptus secretory products in establishment of pregnancy. J Reprod Fert 76: 841-850. doi: 10.5713/ajas.2011.r.08

4. Bravo P, Fowler M, Lasley B, Stabenfeldt G. 1988. Hormonas folículo estimulante y luteinizante en llamas. En: VI Convención Inter sobre Camélidos Sudamericanos. Oruro, Bolivia.

5. Bravo P, Sumar J. 1989. Laparascopic examination of the ovarian activity in alpacas. Anim Reprod Sci 21: 271-281. doi: 10.1016/0378-4320(89)90034-1

6. Bravo P, Stabenfeldt G, Lasley B, Fowler M. 1991. The effect of ovarian follicle size on pituitary and ovarian responses to copulation in domesticated South American camelids. Biol Reprod 45: 553-559.

7. Chipayo Y, Leyva V, García W. 2003. Efecto del estradiol en el periodo de reconocimiento maternal de la preñez sobre la supervivencia embrionaria en alpacas. Rev Inv Vet Perú 14: 24-32. doi: 10.15381/rivep.v14i2.1615

8. Fernández-Baca S, Hansel W, Novoa C. 1970. Corpus luteum function in the alpaca. Biol Reprod 3: 252-261. doi: 10.1093/biolreprod/3.2.252

9. Fernández-Baca S. 1970. Estudio sobre la reproducción de la alpaca (Lama pacos). Bol Ext IVITA Perú 4: 33-42.

10. Fernández-Baca S. 1971. La alpaca, producción y crianza. Lima: IVITA. Bol Divul 7.

11. Fitz T, Mayan M, Sawyer H, Niswen$\operatorname{der} \boldsymbol{G}$ 1982. Characterization of two steroidogenic cells types in the ovine corpus luteum. Biol Reprod 27: 703-711. doi: 10.1095/biolreprod27.3.703

12. Flint A, Sheldrick E. 1983. Evidence for a systemic role for ovarian oxytocin in luteal regression in sheep. J Reprod Fert 67: 215-225. doi: 10.1530/ jrf.0.0670215

13. Flint A, Sheldrick E. 1985. Continous infusion of oxytocin prevents induction of uterine oxytocin receptor and blocks luteal regression in cyclic ewes. J Reprod Fert 75: 623-631. doi: 10.1530/ jrf.0.0750623 
14. Geiser R, Short E, Morgan G 1994. Establishment of pregnancy in domestic farm species. In: Zavy M, Geisert R (eds). Embryonic mortality in domestic species. England: CRC Press. p 23-49.

15. Gilbert C, Lamming G, Parkinson T, Flint A, Wathes D. 1989. Oxytocin infusion from day 10 after estrus extends the luteal phase in non-pregnant cattle. $\mathrm{J}$ Reprod Fert 86: 203-210. doi: 10.1530/ jrf.0.0860203

16. Godkin J, Bazer F, Thatcher W, Roberts R. 1984. Proteins released by cultured day 15-16 conceptuses prolong luteal maintenance when introduced into uterine lumen of cyclic ewes. J Reprod Fert 71: 57-64.

17. Hixon J, Flint A. 1987. Effect of luteolytic dose of oestradiol benzoate on uterine oxytocin receptor concentrations, phosphoinositide turnover and prostanglandin $\mathrm{F}_{2 \alpha}$ secretion in sheep. J Reprod Fert 79: 457-467. doi: 10.1530/ jrf.0.0790457

18. Jarry H, Einspanier A, Kanngierer U, Dietrich M, Pitzel L, Hostz W, Wuttke W. 1990. Release and effects of oxytocin on estradiol and progesterone secretion in porcine corpora lutea as measured by an in vivo microdialysis system. Endocrinology 126: 2350-2358. doi: 10.1210/endo-126-5-2350

19. Leyva V, García W. 1999a. Efecto de la progesterona exógena y endógena en alpacas en celo sobre la ovulación, fertilización y gestación. En: Cong Mundial sobre Camélidos. Cusco, Perú.

20. Leyva V, García W. 1999b. Efecto de la GnRH sobre la fertilización y sobrevivencia embrionaria en alpacas. En: Congreso Mundial sobre Camélidos. Cusco, Perú.

21. Leyva V, García W. 1999c. Efecto de la progesterona exógena sobre la función del cuerpo lúteo de alpacas. En: Congreso Mundial sobre Camélidos. Cusco, Perú.

22. Leyva V, García $\boldsymbol{W}$. 2000. Efecto del estradiol $\left(\mathrm{E}_{2}\right)$ en la fertilización y sobrevivencia embrionaria en alpacas.
XV Congreso Nacional de Ciencias Veterinarias. Cusco, Perú.

23. Leyva V, Franco E, Condorena $N$. 1983. Determinación de la curva lactacional en alpacas y llamas en condiciones de pastura natural. En: VI Reunión Asoc Peruana Prod Anim. Lambayeque, Perú: APPA.

24. Liebermann J, Schams D. 1995. Effects of estradiol-17ß on oxytocin and progesterone release of the bovine corpus luteum during the estrous cycle. $\mathrm{J}$ Reprod Dev 41: 187-196. doi: 10.1262/ jrd.41.187

25. McCracken J, Schramm W, Okulicz W. 1984. Hormone receptor control of pulsatile secretion of $\mathrm{PGF}_{2 \alpha}$ from ovine uterus during luteolysis and its abrogation in early pregnancy. Anim Reprod Sci 7:31-55. doi: 10.1016/0378-4320(84)90027-7

26. Novoa C, Leyva V. 1996. Reproducción en alpacas y llamas. Fondo Colaborador Perú-Suiza, CISA/IVITA. Fac Med Vet, Univ de San Marcos. Serie Pub IVITA $26.30 \mathrm{p}$.

27. Rodgers $R$, O'Shea J, Findlay J, Flint A, Sheldrick E. 1983a. Large luteal cells the source of luteal oxytocin in the sheep. Endocrinology 113: 2302-2304. doi: 10.1210/endo-113-6-2302

28. Rodgers R, O'Shea J, Findlay J, Flint A, Sheldrick E. 1983b. Progesterone production in vitro by small and large ovine luteal cells. J Reprod Fert 69: 113-114.

29. Sheldrick E, Flint A. 1990. Effect of continous infusion of oxytocin on prostaglandin $\mathrm{F}_{2 \alpha}$ secretion and luteolysis in the cyclic ewe. Reprod Fert Develop 2: 89-99.

30. Sumar J, Novoa C, Fernandez-Baca S. 1972. Fisiología reproductiva postpartum en la alpaca. Rev Inv Pec IVITA 1(1): 21-27.

31. Tallam S, Walton J, Johnson W. 2000. Effects of oxytocin on follicular development and duration of the estrus cycle in heifers. Theriogenology 53: 951-962. doi: 10.1016/S0093-691X(00)00242-9 
32. Vallet J, Lamming G, Batten M. 1990. Control of endometrial oxytocin receptor and uterine response to oxytocin by progesterone and oestradiol in the ewe. J Reprod Fert 90: 625-634.
33. Walters D, Schams D, Schallenberger E. 1984. Pulsatile secretion of gonadotrophins, ovarian steroids and ovarian oxytocin during the luteal phase of the oestrus cycle in the cow. J Reprod Fert 71:479-491. 